considerable interval by the interposition of the parietal and alisphenoid. In many inferior races, the interval is greatly diminished, and often, especially among the Melanesians, disappears altogether, the squamosal then directly uniting with the frontal. Very frequently, small independent ossicles, which may be called epipteric bones, as they are placed at the upper end of the great wing of the sphenoid, are interposed. In the Andamanese, the pterion is usually very narrow, but in 6 only out of 46 cases examined (taking both sides) did the squamosal reach the frontal. In 8 cases, epipteric bones were developed. The general surface of the cranium is smooth, and the muscular ridges little pronounced. The forehead is rounded and even, and the glabella and supraorbital eminences are always very little developed. The interspace between the orbits is wide and flat, and there is very little depression at the root of the nose. The orbits are round, the average index, or the ratio of height to width of margin, the latter taken as IOO, being as high as 9ro. The nasal bones have an exceedingly characteristic form, being straight, with nearly parallel sides, and not prominent. The width of the nasal opening, so characteristic of different races, brings the Andamanese into the mesorhine category of Broca, though approaching the platyrhine, the average index being 512 , the maximum 578 , and the minimum 447 . Of the 24 skulls, 5 are platyrhine, 17 mesorhine, and 2 leptorhine (see definitions in Lecture II). With regard to the important character of the projection forward of the jaws compared with the rest of the face, the Andamanese are less prognathous than the Negroes, Tasmanians, and Melanesians, but more so than most other races. Of the 24, I I are decidedly prognathous, 8 mesognathous, and 5 orthognathous, as ascertained by the ready method of comparing the basi-alveolar with the basi-nasal length.

Comparison with other Races.-With the Australians, the Andamanese have very little affinity; the smooth hair of the former entirely separating them, independently of the cranial characters, as dolichocephaly, strongly pronounced brow-ridges, low orbital index, wide nasal aperture, great prognathism, etc. It is to the other woolly-haired races that we must naturally turn in endeavouring to find their nearest relatives. The typical Melanesians and Papuans, as has been before mentioned, differ greatly in their principal cranial characters, especially in the great height and narrowness of the skull. The Tasmanians had wider heads, but their facial characters were more like those of the Australians, and therefore widely different from the Andamanese. The African negroes, again, are almost all dolichocephalic, and, as a general rule, are extremely prognathous, and strongly platyrhine. Many of them, however, have the smooth brow and round orbit seen in the Andamanese, and not generally met with in the true Oceanic negroes.

The natives of the Andaman Islands, with whom may probably be associated the less known Aetas of the Philippines, the Semangs of the Malay peninsula, and some other scattered races of the Indo-Malay peninsula, thus constitute a race apart, to which the name Negrito may properly be applied. At first sight, they appear in their craniological characters to present little affinity to either of the other woolly-haired races, but it is probable that they represent a small or infantile type of the same primary group, as nearly all the characters by which they differ from the other negroes-the smaller size, smoother and more globular heads, absence of supraorbital prominences, rounder orbits, and less projecting jaws, are those which we find in the younger individuals of a species, as compared with the older, or in the smaller species of a natural group as compared with the larger. It is very possible, but this is purely hypothetical, that the Andamanese, whose geographical position is almost midway between either extremes of the range of the woolly-haired races, may be the unchanged or little modified representatives of a primitive type, from which the African negroes on the one hand, and the Oceanic negroes on the other, have taken their origin, and hence everything connected with their history or structure becomes of the greatest interest to the anthropologist.

BEDFORD. - The most remarkable feature in Dr. Prior's report for 1878 is an account of an extraordinarily fatal epidemic of diphtheria, which caused during the year no fewer than fifty-seven deaths. Dr. Prior does not seem to have been able to discover the origin of this outbreak, and we do not learn much as to the circumstances of its spread, except that it was distributed all over the town, and was repeatedly communicated by contagion. Family susceptibility had, as in many other outbreaks, a great deal to do with the attacks. In one house, five children out of a family of seven were swept away; in each of two other houses, three deaths occurred; and in 2 third there were no fewer than eight attacks. None of the other zymotic diszases were very prevalent, except diarrhœa, which caused 33 deaths, 29 being in children under five years of age. The general death-rate was $2 \mathbf{1} .5$ per 1000, which is much higher than that for the preceding year.

\section{AMYLOID DEGENERATION.}

\author{
By GEORGE BUDD, junior.
}

THE term amyloid degeneration has been applied to the infiltration of human tissues with a substance which is in many respects the most curious compound known: a starch, yet yielding nitrogen on analysis; a proteid, perhaps, yet one which resists the action of gastric juice. I say perhaps, for it is the purpose of the present paper to discuss the nature of this compound, and to throw, it is hoped, some light upon its real character. To the term degeneration I still adhere, meaning to suggest, not the retrograde metamorphosis of tissues themselves, but rather the deterioration of some substance concerned normally in the nutritive processes, whose altered state leads to its deposition in the tissues.

The cause of this condition has long remained a matter of doubt. Dickinson regards amyloid substance as identical with dealkalised fibrin. He draws his conclusion mainly from the fact that it occurs commonly in connection with profuse suppuration; but it would be nothing new in the history of medicine if it were some day shown that it was the cause rather than the effect of such pus-formations.

It first appears in the small nutrient arteries, and, after infiltrating their substance, pervades the tissues round about them. This would look as though some substance brought-habitually, perhaps-for the supply of the part had become in a way altered-degenerated, if you will-and deposited at its destination in such a condition that assimilation was no longer possible.

Do we know of any starchy compound concerned commonly in the nutrition of the body upon which we might look as the possible producer of amyloid material? Glycogen would seem to be such a substance. Secreted by the liver and stored up in that organ, it serves as a reserve fund of carbohydrate material for the body-supply. Through what changes it may pass before reaching the tissues, is a matter of doubt. It has been asserted that it becomes converted into sugar; but experiments upon this point have led to strangely opposite results. The stability of this glycogen is liable to great variation. In diabetes artificially produced, it is rendered exceedingly unstable, and is converted into sugar with great readiness. Does it not seem probable that there are other-it may be opposite-influences, such as would engender a contrary condition of things: a condition in which glycogen was rendered exceedingly stable-too stable, in fact, for proper assimilation ? Glycogen in such a state, carried by the blood to its accustomed destination, would there remain, a stable product, assimilated only by the stronger tissues. There it would lie, and there accumulate, blocking up the small nutrient arteries, and impairing or destroying the function of the organ which they supply. Owing to its slow and gradual deposition in a finely divided state, we should expect to find it packing in its substance the remains of the attenuated tissues; giving externally reactions characteristic of glycogen, yet yielding nitrogen on analysis; resisting the action of gastric juice, since the proteids it contains are surrounded by a protective coating of glycogen.*

It will be seen that our predictions would resolve themselves into a terse description of amyloid substance. Like glycogen, it yields, when treated with iodine, a dark mahogany colour; and this reaction is habitually used for its detection. Like glycogen, it is allied to starch, and turns blue when treated with sulphuric acid and iodine.

Analysed by Virchow, it was found, despite its starchy nature, to contain nitrogen: a circumstance which was at one time supposed to depend upon its imperfect separation from surrounding tissues. Virchow's investigations have, however, been confirmed by subsequent analyses whose accuracy cannot be doubted; and I am not aware that the results are explicable on any other hypothesis than the one here set forth.

The following facts deserve notice in addition. Amyloid degeneration occurs most frequently in the liver; that organ is the manufactory and storehouse of glycogen. Cyon and Aladoff consider that the formation of sugar in artificial diabetes is directly dependent upon distension of the hepatic artery and its branches; that the artery, in fact, is intimately connected with the stability of glycogen. Curiously enough, amyloid degeneration always commences in, and is frequently confined to, that zone of the liver-lobule which corresponds to the distribution of the hepatic artery.

In conclusion, I would remark that the occurrence of diabetes and amyloid degeneration in the same individual by no means negatives the theory here laid down; for concentric deposits of phosphates and uric acid-substances indicating opposite conditions of the system-are not unfrequently found in the bladder.

* Such is the case with amyloid material. Any shreds of proteid matter not actually * Such is the case with amic juice, which is commonly used to separate lardacein from the tissues round about. 